Review Article

\title{
The Effect on Treatment Adherence of
} Administering Drugs as Fixed-Dose Combinations versus as
Separate Pills: Systematic Review and Meta-Analysis

\author{
Katy A. van Galen, ${ }^{1,2}$ Jeannine F. Nellen, ${ }^{1}$ and Pythia T. Nieuwkerk ${ }^{2}$ \\ ${ }^{1}$ Division of Infectious Diseases, Tropical Medicine and AIDS, Academic Medical Center, Amsterdam, The Netherlands \\ ${ }^{2}$ Department of Medical Psychology (J3-219-1), Academic Medical Center, Meibergdreef 9, 1105 AZ Amsterdam, The Netherlands
}

Correspondence should be addressed to Pythia T. Nieuwkerk; p.t.nieuwkerk@amc.uva.nl

Received 15 May 2014; Accepted 19 August 2014; Published 4 September 2014

Academic Editor: David Katzenstein

Copyright (C) 2014 Katy A. van Galen et al. This is an open access article distributed under the Creative Commons Attribution License, which permits unrestricted use, distribution, and reproduction in any medium, provided the original work is properly cited.

\begin{abstract}
Administering drugs as fixed-dose combinations (FDCs) versus the same active drugs administered as separate pills is assumed to enhance treatment adherence. We synthesized evidence from randomized controlled trials (RCTs) about the effect of FDCs versus separate pills on adherence. We searched PubMed for RCTs comparing a FDC with the same active drugs administered as separate pills, including a quantitative estimate of treatment adherence, without restriction to medical condition. The odds ratio (OR) of optimal adherence with FDCs versus separate pills was used as common effect size and aggregated into a pooled effect estimate using a random effect model with inverse variance weights. Out of 1258 articles screened, only six studies fulfilled inclusion criteria. Across medical conditions, administering drugs as FDC significantly increased the likelihood of optimal adherence (OR 1.33 (95\% CI, 1.03-1.71)). Within subgroups of specific medical conditions, the favourable effect of FDCs on adherence was of borderline statistical significance for HIV infection only (OR 1.46 (95\% CI, 1.00-2.13)). We observed a remarkable paucity of RCTs comparing the effect on adherence of administering drugs as FDC versus as separate pills. Administering drugs as FDC improved medication adherence. However, this conclusion is based on a limited number of RCTs only.
\end{abstract}

\section{Introduction}

Adherence to combination antiretroviral therapy (cART) is a key predictor of antiretroviral treatment success and survival $[1,2]$. Past research has identified treatment complexity as one of the factors contributing to low levels of adherence [3-6]. The complexity of a patient's medication regimen may refer to the number of prescribed medications per day, that is, pill burden, the daily dosage frequency, and special administration instructions [7]. The vast majority of studies investigating the effect of treatment simplification on adherence have focused on the effect of once daily dosing versus twice daily dosing, generally yielding better adherence rates for once daily regimens $[8,9]$. Another simplification strategy consists of the use of fixed-dose combinations (FDCs). FDCs combine two or more active drugs in one single-tablet or capsule. The reduction in pill burden associated with the use of FDCs is assumed to improve patient adherence. Yet, randomized studies investigating this possible benefit of FDCs are scarce as FDCs are usually approved on the basis of safety and bioequivalence rather than noninferiority to the component regimens.

The question to what extent FDCs lead to better adherence than the same active drugs administered as separate pills has nowadays become highly relevant. The patents of several antiretroviral drugs have recently expired and others will expire in upcoming years. This offers the potential for the replacement of branded FDCs by cheaper generics containing the same active drugs administered as separate pills $[10,11]$. It was recently shown that decoupling of branded FDCs into separate generic and branded drugs could result in considerable cost savings $[12,13]$. Current pressures to control healthcare expenditures in many countries could encourage the decoupling of branded FDCs. Possibly, these cost savings come at the expense of decreased levels of adherence [14]. Knowledge about the quantitative effect on 
adherence of administering drugs as FDCs versus as separate pills of the same active drugs could help making this tradeoff. The objective of the present study was to summarize and synthesize existing research evidence from randomized controlled trials about the effect on treatment adherence of administering drugs as FDCs versus the same active drugs administered as separate pills.

\section{Methods}

We searched the PubMed database from inception to December 2012 for papers fulfilling the following inclusion criteria: (1) randomized controlled trial, (2) comparing a FDC with the same active drugs administered as separate pills, (3) administration route being oral, (4) medications being selfadministered, (5) endpoints of the study included a quantitative estimate of treatment adherence, and (6) the paper being published in English language. We included papers without restriction in type of diseases and/or medications.

We used the following search strategy: (Therapy/ broad[filter]) and (((Fixed-dose combination[tiab] or "Drug Combinations"[Majr] or drug combination* [tiab] or single pill combination* [tiab] or polypill[tiab])) and ("Patient Compliance"[Majr] or compliance* [tiab] or adherence $^{*}\left[\right.$ tiab] or nonadherence ${ }^{*}$ [tiab] or nonadherence* [tiab] or noncompliance ${ }^{*}[$ tiab] or noncompliance* [tiab] or "Treatment Outcome"[Mesh] or treatment outcome* [tiab]))).

If the title and the abstract met inclusion criteria, the full text of the article was retrieved and the article was included if all the inclusion criteria were met. We screened the reference lists of papers identified by our search strategy to find additional potentially eligible studies.

Two authors (KG and PN) independently extracted data from each study that fulfilled inclusion criteria. We extracted the following information: name of the first author, year of publication, sample size, country in which the study was conducted, medical condition for which treatment was prescribed, medicines administered in the intervention group (FDC) and in the control group (separate pills), duration of follow-up, and adherence assessment method.

We used the odds ratio (OR) of optimal adherence with FDCs versus separate pills as common effect size. We calculated the $I^{2}$ index as measure of between study heterogeneity in effect sizes. We used a random effect model with inverse variance weights to aggregate individual effect sizes into a pooled effect estimate with $95 \%$ confidence limits using Review Manager 5.2. We repeated this analysis for subgroups of different medical conditions. Additionally, we repeated the analysis with the study on hypertension being removed from the analysis. We examined the presence of possible publication bias by visual inspection of funnel plots and by investigating the statistical significance of Egger's regression intercept using Comprehensive Meta-Analysis version 2.

\section{Results}

A total of 1258 potentially relevant articles were identified and were subsequently screened (Figure 1). A total of 16 full text articles were assessed for eligibility. Of these, ten articles were excluded for the following reasons: medicines were not self-administered $(n=6)$, separate pills had not the same active drugs as FDCs $(n=3)$, and the study design was not a randomized controlled trial $(n=1)$. The characteristics of the 6 included studies are shown in Table 1.

Results of the quantitative pooling of effect sizes are shown in Figure 2. Across medical conditions, administering drugs as FDC versus as separate pills significantly increased the likelihood of optimal adherence. Within subgroups of specific medical conditions, the favorable effect of FDCs versus separate pills on adherence was statistically significant for HIV infection only, although results for TB and hypertension were in the same direction. After removing the study on hypertension, administering drugs as FDC still significantly increased the likelihood of optimal adherence (OR 1.31 (95\% CI, 1.00-1.73)). Neither the funnel plot nor Eggers regression intercept $(P=0.16)$ was indicative of publication bias (data not shown).

\section{Discussion}

In our meta-analysis of randomized controlled trials comparing the effect on treatment adherence of administering drugs as FDCs versus as separate pills, FDCs resulted in improved adherence compared with separate pills. Nevertheless, the result was based on a limited number of studies only, as we observed a remarkable paucity of RCTs comparing the effect on adherence of administering drugs as FDC versus as separate pills. Across various medical conditions, this effect of FDCs on adherence was statistically significant for the treatment of HIV infection only, although the effect for other medical conditions was in the same direction.

Our results are in line with a previous meta-analysis investigating the effect of fixed dose combinations on medication compliance [21]. This previous meta-analysis found a substantial reduction in the risk of nonadherence for FDCs compared with non-FDCs. Results from the present study are in the same direction but are less pronounced. This previous meta-analysis included not only randomized controlled trials, but also retrospective observational studies. Nonrandomized studies have been shown to overestimate intervention effects [22]. When the previous meta-analysis was restricted to randomized studies, the favourable effect of FDCs diminished and was no longer statistically significant. Nonrandomized studies are more susceptible to unaccounted confounding than randomized studies. Combining evidence from randomized and nonrandomized studies studies is not recommended [23]. We therefore included only randomized trials in the present meta-analysis.

A number of observational studies in HIV infection have compared adherence and/or virological response with single versus multitablet regimens [24-28]. In one study, switching patients from multitablet regimens to single-tablet regimens resulted in improved adherence while maintaining virological response [24]. In another study, switching patients from single-tablet regimens to multitablet regimens did not result in a diminished virological response [25]. Some studies comparing adherence and/or virological response among patients on single versus multitablet regimens have found 


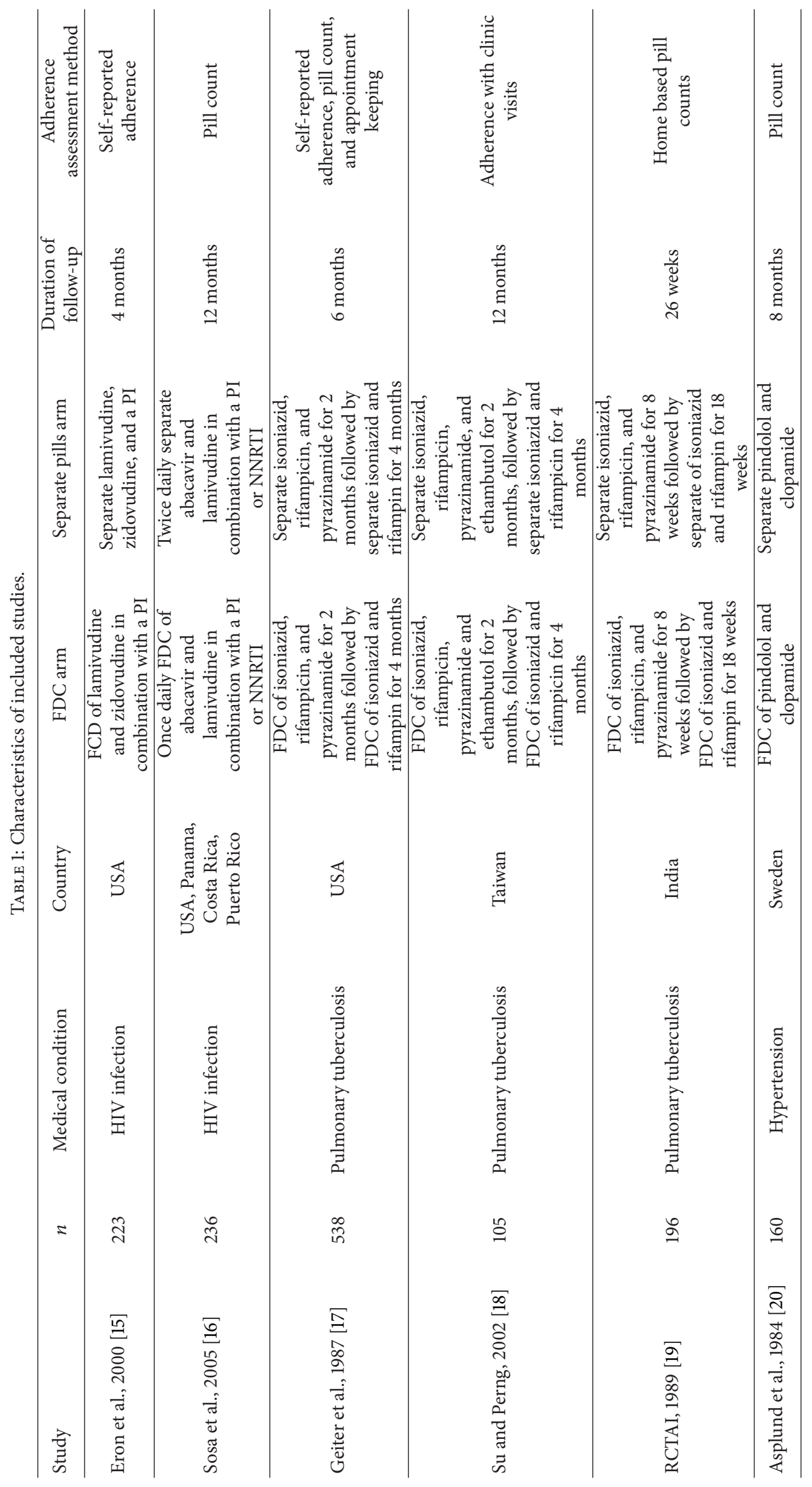




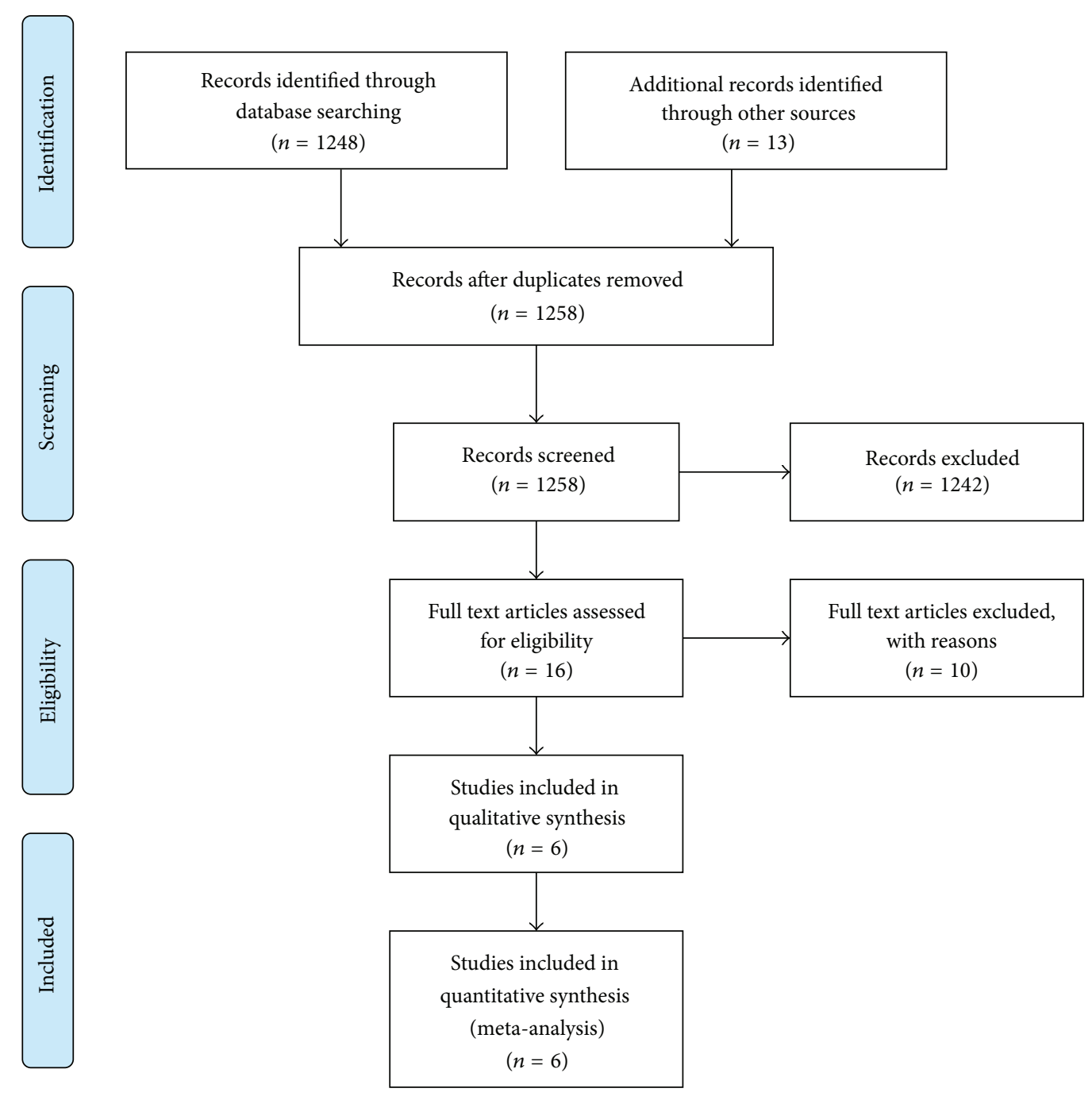

FIGURE 1: Flow diagram.

significantly higher adherence $[26,27]$ and/or a more favorable virological response in the single-tablet groups $[26,28]$ compared with the multitablet groups, whereas others have found no difference in adherence [29].

Studies investigating the association between daily pill burden and virological response have demonstrated a significant relationship between a higher pill burden and a lower virological suppression rate $[30,31]$ that was independent of daily dosing frequency [31]. Most of these observational studies suggest that single-tablet regimens result in higher levels of adherence and improved virological response. However, interpretation of the results of these studies is complicated by methodological limitations. The switch studies did not include a comparison group. All of the observational studies may have been susceptible to unmeasured confounding in particular channelling bias. Because the selection of treatments was not random in these studies and was most likely determined by patient and physician characteristics, the observed effects of single-tablet regimens in comparison with multitablet regimens may have been influenced by factors other than the treatment. For example, physician may be more likely to prescribe multitablet boosted protease inhibitor based regimens to patients with higher baseline viral load or those judged less likely to be adherent, as these drugs are generally more "forgiving" of missed doses. Another example is that patients with prior virological treatment failure may be more likely to be prescribed multitablet regimens and to have higher viral loads.

The present study adds to these previous studies that evidence from randomized controlled trials of FDCs versus separate pills also show improved levels of adherence for FDCs. The present study has several limitations. First, the number of randomized controlled trials comparing FDCs with separate pills is small. Therefore, we were able to include only a limited number of trials in our meta-analysis and we choose to include trials irrespective of the medical condition for which treatments were prescribed. Second, for one of the trials conducted in HIV infection it was impossible to distinguish the effect of FDCs from the effect of daily dosing frequency [16]. Third, we searched for papers in the PubMed database 


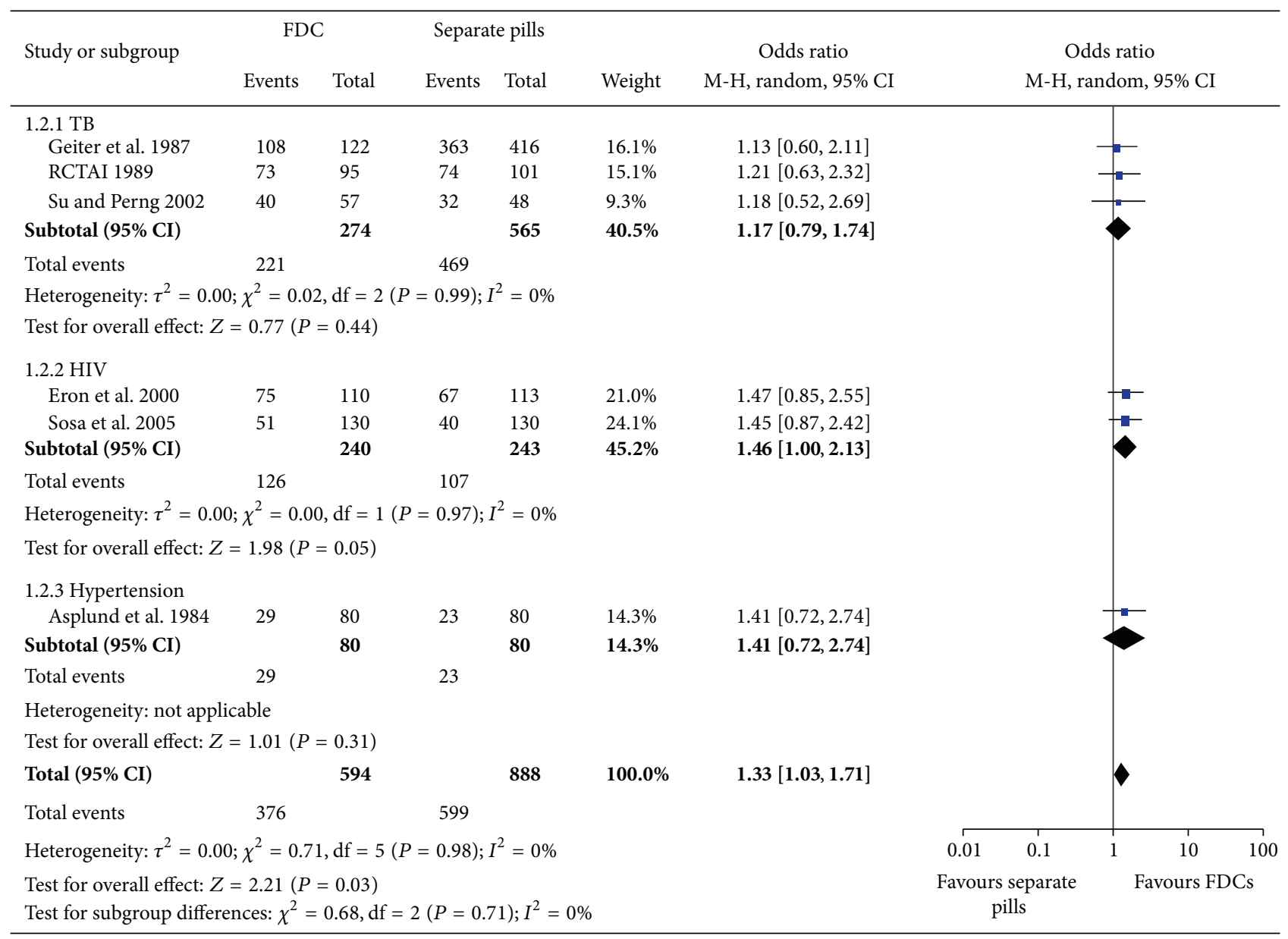

FIGURE 2: Effect of FDCs versus separate pills on treatment adherence.

only and restricted our search to full text papers. Fourth, studies included in our meta-analysis have used a variety of adherence assessment methods. Nevertheless, the statistical heterogeneity between studies in effect sizes was low.

In conclusion, our meta-analysis of randomized controlled trials yielded an increased likelihood of improved adherence for FDCs compared with separate pills. However, this conclusion is based on a limited number of randomized controlled trials.

Our findings suggest that decoupling FDCs into separate pills could lead to lower levels of adherence. We, therefore, recommend careful monitoring of patients who are switched from FDCs to multitablet regimens for economic reasons.

\section{Conflict of Interests}

The authors declare that there is no conflict of interests regarding the publication of this paper.

\section{References}

[1] S. Mannheimer, G. Friedland, J. Matts, C. Child, and M. Chesney, "The consistency of adherence to antiretroviral therapy predicts biologic outcomes for human immunodeficiency virus-infected persons in clinical trials," Clinical Infectious Diseases, vol. 34, no. 8, pp. 1115-1121, 2002.

[2] V. D. Lima, R. Harrigan, D. R. Bangsberg et al., "The combined effect of modern highly active antiretroviral therapy regimens and adherence on mortality over time," Journal of Acquired Immune Deficiency Syndromes, vol. 50, no. 5, pp. 529-536, 2009.

[3] T. R. Glass, M. Battegay, M. Cavassini et al., "Longitudinal analysis of patterns and predictors of changes in self-reported adherence to antiretroviral therapy: swiss HIV cohort study," Journal of Acquired Immune Deficiency Syndromes, vol. 54, no. 2, pp. 197-203, 2010.

[4] C. Protopopescu, F. Raffi, P. Roux et al., "Factors associated with non-adherence to long-term highly active antiretroviral therapy: a 10 year follow-up analysis with correction for the bias induced by missing data," Journal of Antimicrobial Chemotherapy, vol. 64, no. 3, pp. 599-606, 2009.

[5] M. Lazo, S. J. Gange, T. E. Wilson et al., "Patterns and predictors of changes in adherence to highly active antiretroviral therapy: longitudinal study of men and women," Clinical Infectious Diseases, vol. 45, no. 10, pp. 1377-1385, 2007.

[6] A. Ammassari, M. P. Trotta, R. Murri et al., "Correlates and predictors of adherence to highly active antiretroviral therapy: overview of published literature," Journal of Acquired Immune 
Deficiency Syndromes, vol. 31, supplement 3, pp. S123-S127, 2002.

[7] V. E. Stone, J. Jordan, J. Tolson, R. Miller, and T. Pilon, "Perspectives on adherence and simplicity for HIV-infected patients on antiretroviral therapy: self-report of the relative importance of multiple attributes of highly active antiretroviral therapy (HAART) regimens in predicting adherence," Journal of Acquired Immune Deficiency Syndromes, vol. 36, no. 3, pp. 808816, 2004.

[8] J. B. Nachega, M. J. Mugavero, M. Zeier, M. Vitória, and J. E. Gallant, "Treatment simplification in HIV-infected adults as a strategy to prevent toxicity, improve adherence, quality of life and decrease healthcare costs," Patient Preference and Adherence, vol. 5, pp. 357-367, 2011.

[9] J.-J. Parienti, D. R. Bangsberg, R. Verdon, and E. M. Gardner, "Better adherence with once-daily antiretroviral regimens: a meta-analysis," Clinical Infectious Diseases, vol. 48, no. 4, pp. 484-488, 2009.

[10] B. Gazzard, C. Moecklinghoff, and A. Hill, "New strategies for lowering the costs of antiretroviral treatment and care for people with HIV/AIDS in the United Kingdom," ClinicoEconomics and Outcomes Research, vol. 4, no. 1, pp. 193-200, 2012.

[11] M. Stoll, C. Kollan, F. Bergmann et al., "Calculation of direct antiretroviral treatment costs and potential cost savings by using generics in the German HIV ClinSurv Cohort," PLoS ONE, vol. 6, no. 9, Article ID e23946, 2011.

[12] G. L. Colombo, S. di Matteo, and F. Maggiolo, "Antiretroviral therapy in HIV-infected patients: a proposal to assess the economic value of the single-tablet regimen," ClinicoEconomics and Outcomes Research, vol. 5, no. 1, pp. 59-68, 2013.

[13] R. P. Walensky, P. E. Sax, Y. M. Nakamura et al., "Economic savings versus health losses: the cost-effectiveness of generic antiretroviral therapy in the United States," Annals of Internal Medicine, vol. 158, no. 2, pp. 84-92, 2013.

[14] J. M. Llibre, J. R. Arribas, P. Domingo et al., "Clinical implications of fixed-dose coformulations of antiretrovirals on the outcome of HIV-1 therapy," AIDS, vol. 25, no. 14, pp. 1683-1690, 2011.

[15] J. J. Eron, E. S. Yetzer, and P. J. Ruane, "Efficacy, safety, and adherence with a twice-daily combination lamivudine/zidovudine tablet formulation, plus a protease inhibitor, in HIV infection," AIDS, vol. 14, no. 6, pp. 671-681, 2000.

[16] N. Sosa, C. Hill-Zabala, E. DeJesus et al., "Abacavir and lamivudine fixed-dose combination tablet once daily compared with abacavir and lamivudine twice daily in HIV-infected patients over 48 weeks (ESS30008, SEAL)," Journal of Acquired Immune Deficiency Syndromes, vol. 40, no. 4, pp. 422-427, 2005.

[17] L. J. Geiter, R. J. O’Brien, D. L. Combs, and D. E. Snider Jr., "United States Public Health Service Tuberculosis Therapy Trial 21: preliminary results of an evaluation of a combination tablet of isoniazid, rifampin and pyrazinamide," Tubercle, vol. 68, no. 2, pp. 41-46, 1987.

[18] W.-J. Su and R.-P. Perng, "Fixed-dose combination chemotherapy (Rifater/Rifinah) for active pulmonary tuberculosis in Taiwan: a two-year follow-up," International Journal of Tuberculosis and Lung Disease, vol. 6, no. 11, pp. 1029-1032, 2002.

[19] Research Committee of the Tuberculosis Association of India, "Fifth tuberculosis association of India: short course chemotherapy trial," Indian Journal of Tuberculosis, vol. 36, no. 2, pp. 95-101, 1989.
[20] J. Asplund, M. Danielson, and P. Ohman, "Patients compliance in hypertension-the importance of number of tablets," British Journal of Clinical Pharmacology, vol. 17, no. 5, pp. 547-552, 1984.

[21] S. Bangalore, G. Kamalakkannan, S. Parkar, and F. H. Messerli, "Fixed-dose combinations improve medication compliance: a meta-analysis," The American Journal of Medicine, vol. 120, no. 8, pp. 713-719, 2007.

[22] J. P. A. Ioannidis, A.-B. Haidich, M. Pappa et al., "Comparison of evidence of treatment effects in randomized and nonrandomized studies," The Journal of the American Medical Association, vol. 286, no. 7, pp. 821-830, 2001.

[23] B. C. Reeves, J. J. Deeks, J. P. T. Higgins, and G. A. Wells, "Including non-randomized studies," in Cochrane Handbook for Systematic Reviews of Interventions Version 5.1.0, J. P. T. Higgins and S. Green, Eds., chapter 13, 2011.

[24] M. Airoldi, M. Zaccarelli, L. Bisi et al., "One-pill once-a-day HAART: a simplification strategy that improves adherence and quality of life of HIV-infected subjects," Patient Preference and Adherence, vol. 4, pp. 115-125, 2010.

[25] F. Engsig, J. Gerstoft, M. Helleberg, G. Kronborg, L. Mathiesen, and N. Obel, "Virologica 1 response in patients who for economic reasons were changed from Atripla to a multitablet cART regimen," in Proceedings of the 20th Conference on Retroviruses and Opportunistic Infections (CROI '13), Atlanta, Ga, USA, March 2013, Abstract 579.

[26] G. A. Rao, S. S. Sutton, J. Hardin et al., "Impact of highly active antiretroviral therapy regimen on adherence and risk of hospitalization in veterans with HIV/AIDS," in Proceedings of the 53rd Interscience Conference on Antimicrobial Agents and Chemotherapy (ICAAC '13), Denver, Colo, USA, September 2013, Abstract H-1464.

[27] C. J. Cohen, J. L. Meyers, and K. L. Davis, "Association between daily antiretroviral pill burden and treatment adherence, hospitalisation risk, and other healthcare utilisation and costs in a US Medicaid population with HIV," BMJ Open, vol. 3, Article ID e003028, 2013.

[28] S. Kapadia, R. Grant, and S. Hodder, "Virologic response better with single tablet fixed dose antiretroviral regimens compared with multiple tablet regimens in an urban population of HIVinfected persons," in Proceedings of the IDWeek, Advancing Science, Improving Care, Abstract 168, San Francisco, Calif, USA, October 2013.

[29] A. Buscher, C. Hartman, M. A. Kallen, and T. P. Giordano, "Impact of antiretroviral dosing frequency and pill burden on adherence among newly diagnosed, antiretroviral-naïve HIV patients," International Journal of STD and AIDS, vol. 23, no. 5, pp. 351-355, 2012.

[30] J. A. Bartlett, R. DeMasi, J. Quinn, C. Moxham, and F. Rousseau, "Overview of the effectiveness of triple combination therapy in antiretroviral-naive HIV-1 infected adults," AIDS, vol. 15, no. 11, pp. 1369-1377, 2001.

[31] J. B. Nachega, J. J. Parienti, O. A. Uthman et al., "Lower pill burden and once-daily antiretroviral treatment regimens for hiv infection: a meta-analysis of randomized controlled trials," Clinical Infectious Diseases, vol. 58, pp. 1297-1307, 2014. 


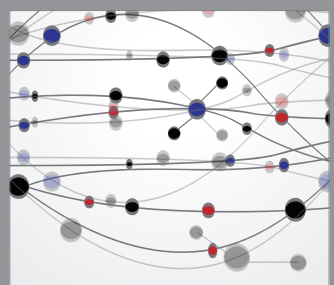

The Scientific World Journal
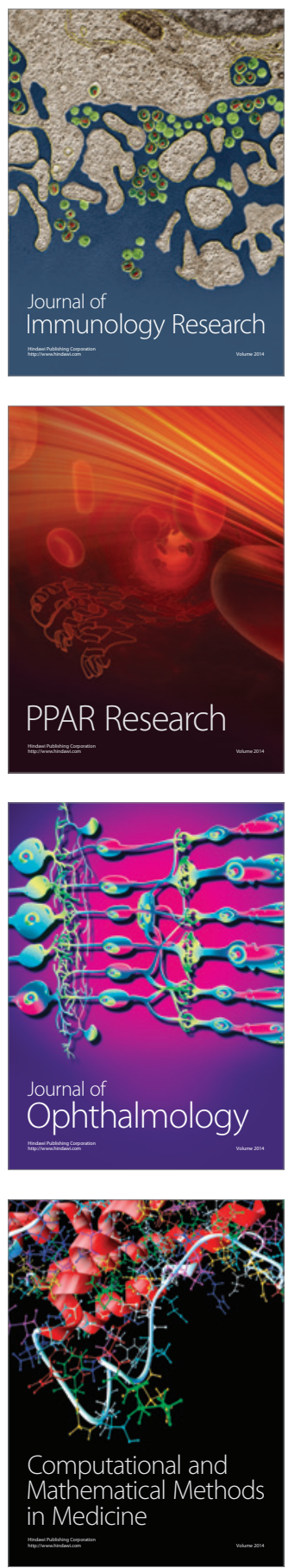

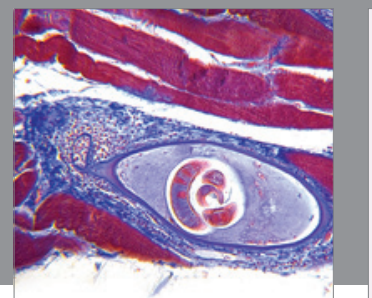

Gastroenterology

Research and Practice
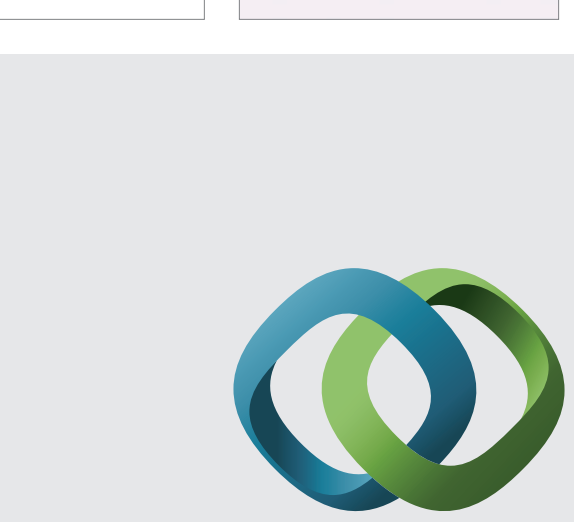

\section{Hindawi}

Submit your manuscripts at

http://www.hindawi.com
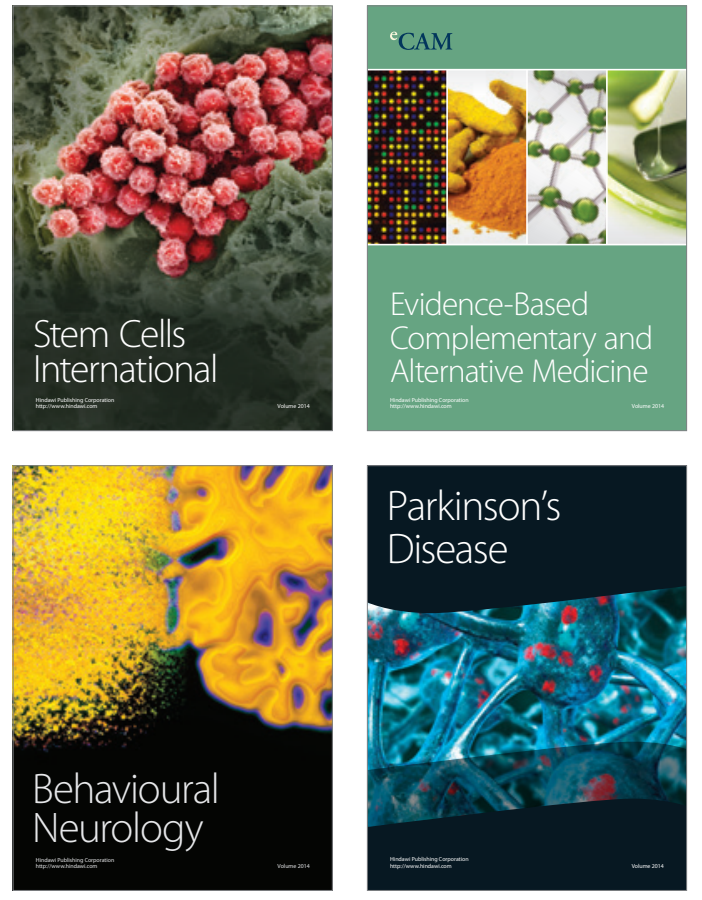
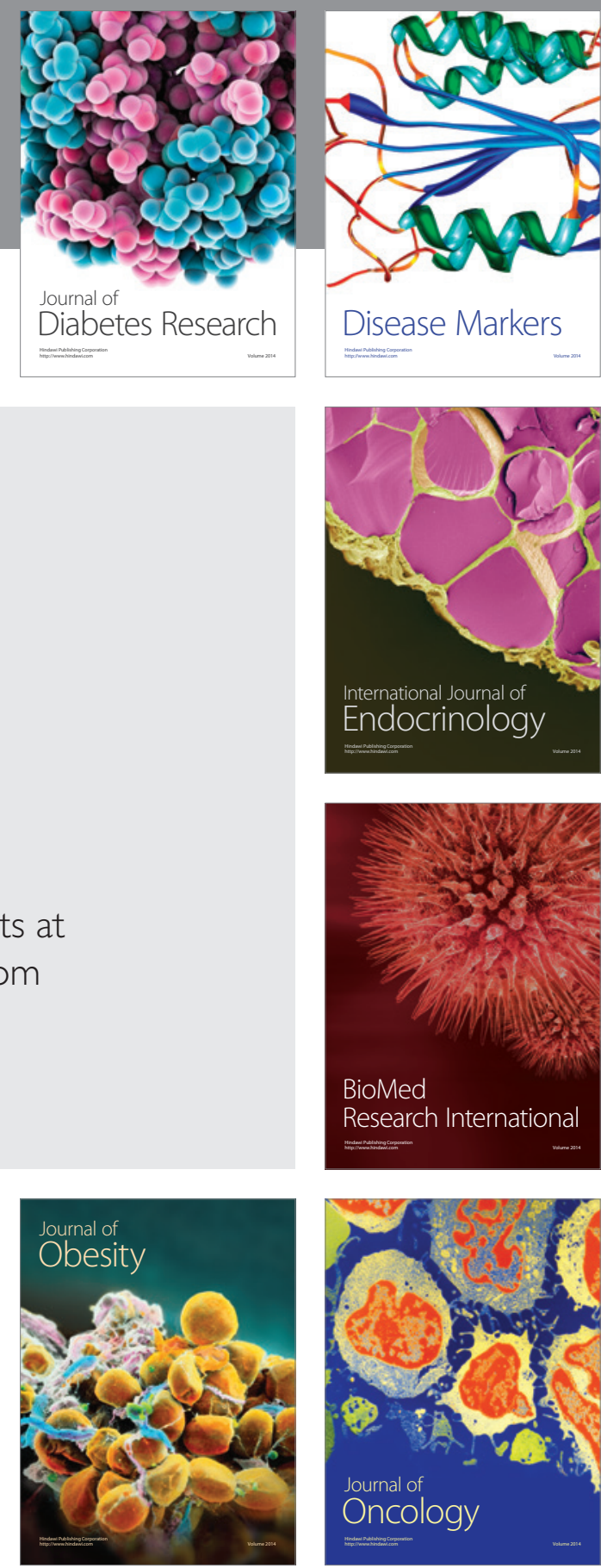

Disease Markers
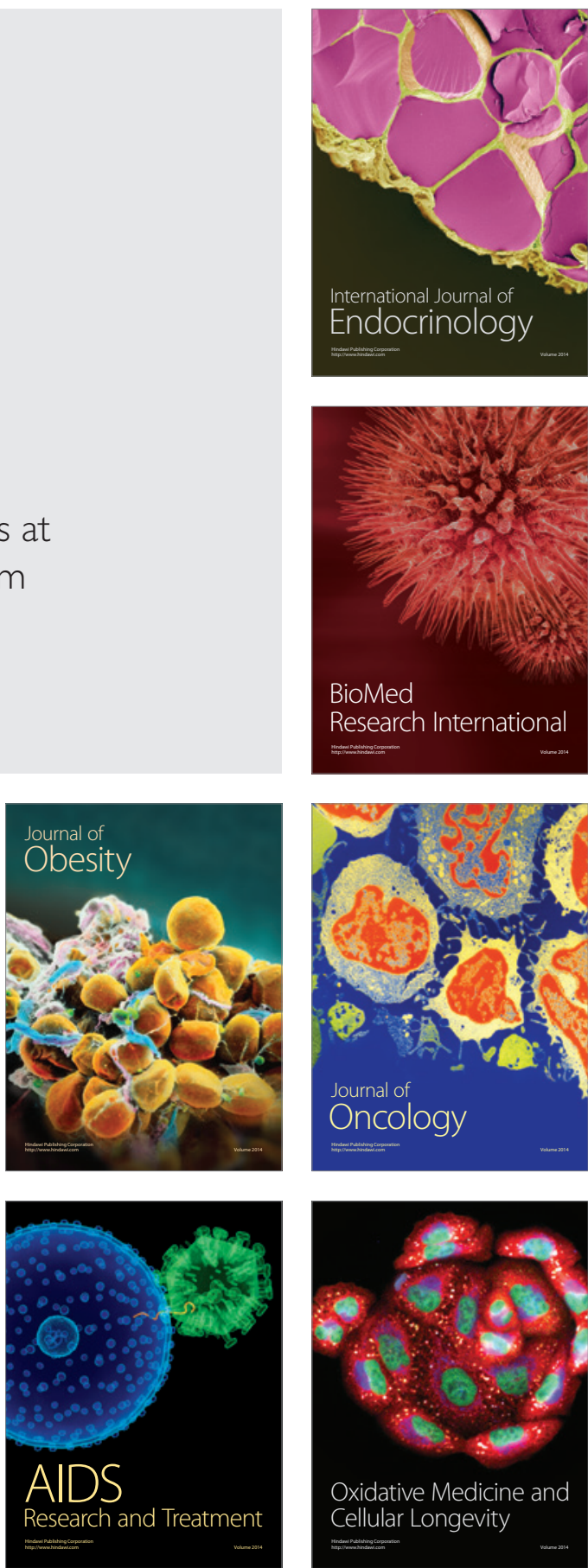